\title{
Automated XPS Analysis of Passivated Stainless Steel to the SEMI Standard
}

\author{
R. G. White*, T. S. Nunney*, B. R. Strohmeier**, J. D. Piasecki**, and R. J. Lee** \\ * Thermo Fisher Scientific, Birches Ind. Est. Imberhorne Lane, East Grinstead, West Sussex, \\ RH19 1UB, UK
}

** RJ Lee Group, Inc., 350 Hochberg Road, Monroeville, PA 15146, USA

The Semiconductor Equipment Manufacturers Institute (SEMI) defines methods for analysing the elemental composition and metallurgical characteristics of metal used in components for high purity gas distribution systems. Typically stainless steel components are treated to improve surface quality and prolong their lifetime and performance. In the passivation process a chromium-rich 'passive' layer is formed on top of the steel surface. The SEMI procedures define methods to determine the surface and oxide composition and chemistry of the interior surface of chromium enhanced stainless steel tubing. The key metrics that are used to identify passivated steel are the total $\mathrm{Cr} / \mathrm{Fe}$ surface atomic ratio, the $\mathrm{Cr}$ oxide/Fe oxide surface atomic ratio, and the total oxide thickness.

The current SEMI test methods have been used since 1992 (former SEMASPEC methods) and use both X-ray Photoelectron Spectroscopy (XPS) and Auger Electron Spectroscopy (AES) to evaluate the passivation of the stainless steel surface. Since then XPS instrumentation has developed significantly which enables the determination of the parameters quickly and easily with XPS only. This work describes how the test procedure can be automated from start to finish with combination of modern XPS instrumentation and software. In particular, procedures can be used to maintain consistency of approach in the important peak-fitting steps to eliminate inconsistencies which can be introduced by different users. The automated approach can be extended to include sputter depth profiling of the passivation layer, resulting in a method for batch control or failure analysis of a series of samples. 

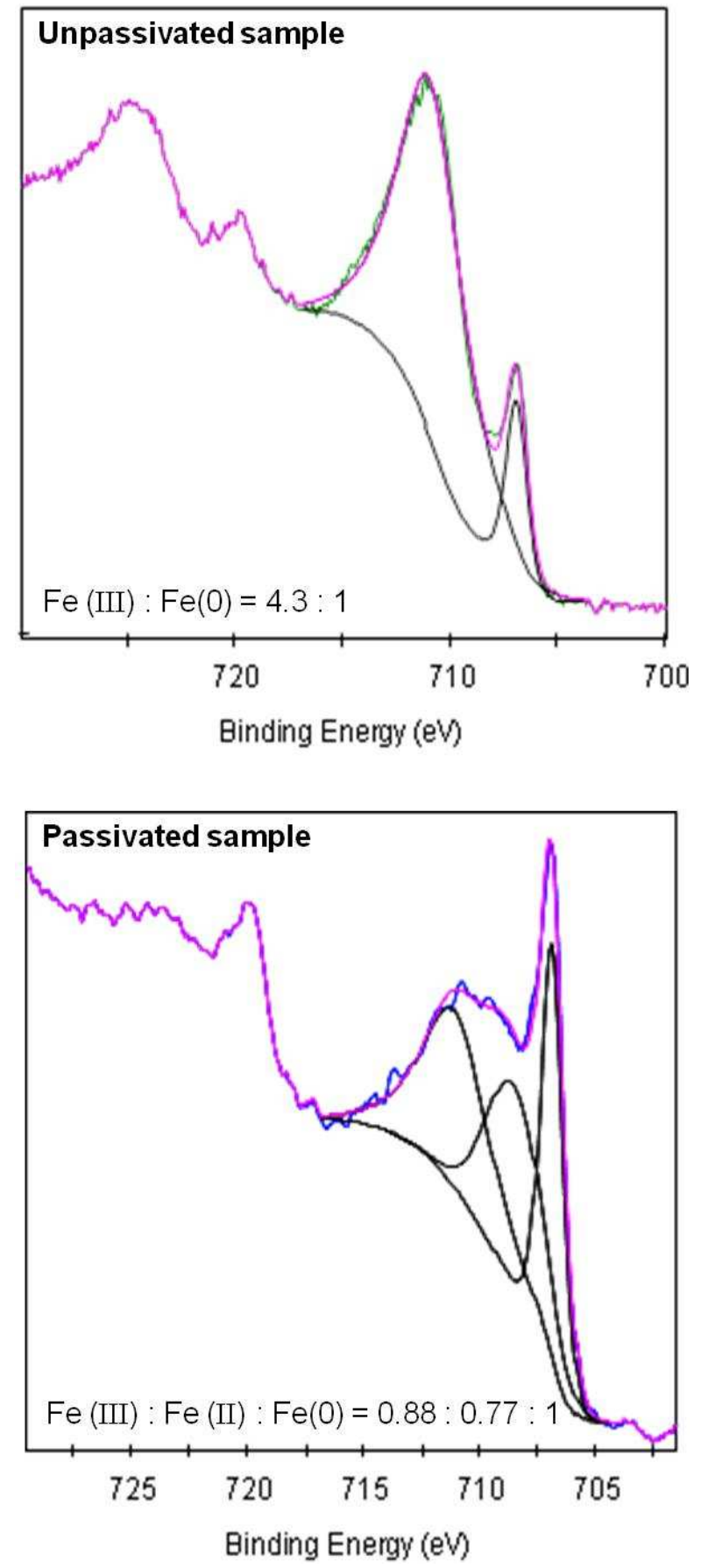

Fig 1. X-ray photoelectron spectra of the $\mathrm{Fe} 2 \mathrm{p}$ region, showing the difference between passivated and unpassivated stainless steel. The concentration of oxide present can be measured directly from the data, and used to calculate the oxide thickness on the sample. 